Anatomical and Phytochemical Study of Glossostemon bruguieri

(Desf.) Sterculiaceae in Kurdistan Region of Iraq

Khalid Faiq Darweesh

Karzan Mahmood Ahmed

\title{
Anatomical and Phytochemical Study of Glossostemon bruguieri (Desf.) Sterculiaceae in Kurdistan Region of Iraq
}

\author{
Khalid Faiq Darweesh ${ }^{1}$ and Karzan Mahmood Ahmed ${ }^{2}$ \\ ${ }^{1}$ Dept. of Biology- Faculty of education- Univ.of Garmian- Kurdistan, Iraq \\ ${ }^{2}$ Dept. of Chemistry- Faculty of education- Univ.of Garmian- Kurdistan, Iraq
}

Received 4 January 2016

Accepted 26 April 2016

\begin{abstract}
$\underline{\text { Abstract }}$
Glossostemon bruguieri named "ARAB QŌZI" or "MOGHAT", which is used in the traditional medicine for treatment various ailments, where no such study has been conducted so far. This investigations report for the first time the anatomical characters and identify the chemical constituents of methanolic extract for different parts of G. bruguieri by using modern sensitive gas chromatography - mass spectroscopy (GC-MS).The microscopic study showed the present of internal duct in all studied parts, also present of oil drops in the cells of root ,cells with special bodies near the vascular bundle of leaves and its petioles and the present of (Stellate, dendroid and multicellular glandular) trichome in aerial parts of the plant. GC-MS analysis revealed the presence of many compounds in different parts of G. bruguieri different between the parts in relation to the anatomical structure which reflex the importance of the aerial parts of the plant in addition to traditional use of root.
\end{abstract}

Keywords: Glossostemon bruguier (Moghat), anatomy, phytochemical analysis. 
Anatomical and Phytochemical Study of Glossostemon bruguieri

(Desf.) Sterculiaceae in Kurdistan Region of Iraq

Khalid Faiq Darweesh

Karzan Mahmood Ahmed

\title{
دراسة تشريحية كيمياوية للنوع Glossostemon bruguieri (Sterculiaceae) النامية في اقليم كوردستان العراق \\ خالد فائق درويش 1 و كارزان محمود احمد2 \\ 1 قسم علوم الحياة ـ فاكلتي التربية - جامعة كرميان - كوردستان - عراق.

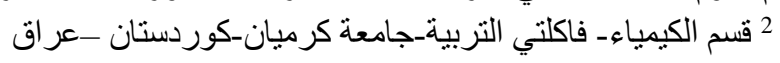

\begin{abstract}
الخلاصة
النوع (Glossostemon bruguieri (Desf.) المعروف محلياً بعرب قوزي يستخدم بصورة تقليدية لعلاج العديد من

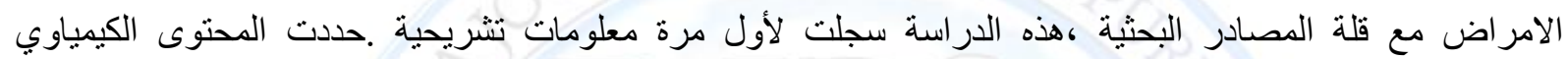
للمستخلص الميثانولي لاجز اء مختلفة من النبات G. bruguieri بأستخدام كروماتو غر افيا الحالة الغازية ومطياف الكتلة الحساسة (GC-MS) .الدراسة التشريحية اظهرت وجود قنوات افرازية في جميع الاجزاء الددروسة وظهور قطرات زيتية في خلايا الجذر مع وجود خلايا ذات تر اكيب خاصة قرب الحزمة الوعائية للورقة واعنقها ووجود شعير ات نجمية وشجرية و غدية متعددة الخلايا في بشرة الاجز اء الهو ائية. التحليل الكيمياوي باستخدام (GC-MS) للمستخلص الميثانولي كثفت عن وجود مركبات عديدة في الازهار والاوراق و النورات الزهرية والساق للنوع Gruguieri مختلفة تبعا للفروقات التشريحية التي اظهرت اهمية الساق بالاضافة الى الاستخدامات التقليدية للجذر. كلمات مفتاحية : Glossostemon bruguier، مغات ،تشريح ،تحليل كيمياوي
\end{abstract}

\section{Introduction}

Despite that Sterculiaceae have no long reputation as medicinal plant family also no high variety of taxa, some species such as moghat (ARAB QŌZI was the common name) Glossostemon bruguieri which is the only native genus and species of this family in Iraq (Townsend and Evan , 1980) have a history in ethnobotany . G. bruguieri is native to Iraq and Iran, and was cultivated in Egypt for its edible roots a long time ago (Meselhy, 2003), distributed in Upper Jazira ,Central Alluvial Plain , Nineveh, Kirkuk and Persian foothills districts of Iraq (Townsend and Evan,1959). (Al-Rawi and Chakravarty, 1964) refers to root of G. bruguieri as medicinal plant with aphrodisiac uses and tonic against cough. (Ibrahim etal., 1997) Evaluate the content and composition of proteins and mucilage of the roots and seeds of Moghat, while (El-Sayed et al, 2004) study the Phytochemical content which show characters of new flavonoids and the effect of aerial parts of G. bruguieri on urine volume. 
Anatomical and Phytochemical Study of Glossostemon bruguieri

(Desf.) Sterculiaceae in Kurdistan Region of Iraq

Khalid Faiq Darweesh

Karzan Mahmood Ahmed

(El-Kiey and Hashem, 1957) study Pharmacognostical importance of Moghat in Egypt this refer to its importance as food and medicinal additives. The structures and the Occurrence of some compound such as 4-methoxyisoscutellargin, sesamin, chrysophanol, emodin and methoxyemodin (physician) and the new compound (3"'-hydroxycupressuflavone) in Moghat reported for the first time by (Meselhy, 2003). Mucilage from G. bruguieri roots exerted a pronounced hypoglycemic action bringing the glucose level down to half (Eddouks and Zeggwagh, 2014). The root extract of G. bruguieri showed no activity at all for all tested bacteria used in study of antibacterial potentiality of some edible plant by (El-Sayed etal., 2014) whereas variety of antibacterial and antifungal activities of $G$. bruguieri reported by (Zain etal., 2014), Also mentioned by (Sher and Alyemeni, 2011) as medicinal plants used in Ethnoveterinary practices in Saudi Arabia. With these uses and interesting of this species there is no anatomical and phytochemical study of other parts of plant in compare with the limited research on root and seeds, also no data about species status, for that we investigate about structure and chemical constituents in different plant parts which make this plant to be deliberated also check about species status.

\section{Material and Methods}

\section{Plant collection}

Survey and checking about the species in remained studies to collection and evaluation of species status, some data obtained by traveling in the field and because of low safety in some area others data collected by contacting herbalist and botanist in referred physiographic districts in Iraq (plate 1 B), Whole parts of Glossostemon bruguieri were collected beside road between Kirkuk and Kalar in Kurdistan region during Jun 2015. The plant material was identified and classified by National Herbarium-Baghdad/Iraq. The collected samples were separated and packed directly in polyethylene bugs. The flower, leaf, stem and inflorescence plant parts were cut and washed with tab water in order to remove dust. The samples were dried under shade at room temperature until they reached a constant weight, and then powdered finely by using grinder machine (IKA-WERKE-M20-Germany) for 30 second. Consequently the dry plant samples were pulverized into powdered form, and stored in dark 
Anatomical and Phytochemical Study of Glossostemon bruguieri

(Desf.) Sterculiaceae in Kurdistan Region of Iraq

Khalid Faiq Darweesh
Karzan Mahmood Ahmed

condition. Other parts preserved in F.A.A solution (Formalin, Acetic acid and Alcoholic ethanol) for anatomical study.

\section{Anatomical Investigation:}

The different parts of the present species was collected and preserved in F.A.A solution, cross sections prepared directly by Razor blade as hand free section or by Freezing microtome (SLEE Model $m t c-G e r m a n y)$, to show different types of cells and tissue systems, samples stained with Safranin $\mathrm{O}$ and sometimes by logules solution to check about the starchy and special cells (Chemicals provided by Sharlu of Spain). Prepared sections examined by light microscope (Meiji 4300L, Japan)and some time by stereo microscope (Meiji RZ model, Japan), prepared slides documented by Canon Camera Kiss model, the specialized cells and tissues as ducts investigated in different parts of the plants.

\section{Preparation of methanolic extracts}

$10 \mathrm{gm}$ of aerial parts of plants (leaf, stem, flower and inflorescence) was extracted with methanol solvent $(100 \mathrm{~mL})$ by maceration extraction for 6 hours under mechanical stirring at room temperature. The procedure was repeated three times. After extraction, it was filtered and the methanol solvent was evaporated by using rotary evaporation (Laborota 4000, Heidolph Instruments, Schwabach, Germany, temperature $40-45^{\circ} \mathrm{C}$ ). The obtained extracts were stored at room temperature for further studies.

\section{GC/MS Analysis}

The protocols were experimentally tested and designed ,the methanolic extraction of aerial parts of plants was analyzing by using GC-MS. Shimadzu Model QP-2010 Mass spectrometer under the following conditions: HP-5 MS (5\% phenylmethylsiloxane) capillary column $(30 \mathrm{~m} \times 0.25 \mathrm{~mm}$ i.d., film thickness $0.25 \mu \mathrm{m})$. Inert gas of helium was used as a carrier gas at constant flow rate of $1.61 \mathrm{ml} / \mathrm{minute}$. Injection port temperature and interface temperature were set at 230 and $245^{\circ} \mathrm{C}$ respectively. Ion Source Temperature was $250^{\circ} \mathrm{C}$. Initial column temperature was $60^{\circ} \mathrm{C}$, held for 2 minute and increased at $70^{\circ} \mathrm{C} / \mathrm{min}$ to $305^{\circ} \mathrm{C}$ and held for $5 \mathrm{~min}$. An electron ionization system with ionization energy $70 \mathrm{eV}$ was used for the detection of compounds. $75 \mathrm{mg}$ of Methanol leaf extraction was taken and made up to 15 $\mathrm{ml}$ with methanol, from which $1 \mu \mathrm{l}$ of sample was automatically injected (split mode) in the 


\section{Anatomical and Phytochemical Study of Glossostemon bruguieri}

(Desf.) Sterculiaceae in Kurdistan Region of Iraq

Khalid Faiq Darweesh

Karzan Mahmood Ahmed

column and mass spectral scan range was set at 45-500 amu. The split ratio was of 1:15. The mass spectrum of the unknown component was compared with the spectrum of the known components stored in the Wiley library. The name, molecular weight, and structure of the components of the test material were finally ascertained.

\section{Results and Discussion}

\section{Species status and anatomical study:}

Despite that project of flora of Iraq was neglected in the last years but it has been that some plants as studied species lost it is density this may be because of over harvesting by herbalist or Egyptian people in seventeen's and eighteens of last century or because of global warming which causing elevation in atmospheric temperature as our observations since 2011 (plate 1, A ) also some area as Jabal Singar undergo flooding in different years which noticed by collagenous researchers among different sites we recorded the best one of them between kifri and Kalar city (Garmin GPS72: N 34 ${ }^{\circ} 43.391^{\prime}$ E04507.020' Elevation: 338.5). For that we suggest that the conservation status of this species at least not evaluated (NE) or me be near threatened (NT) (IUCN, 2012).
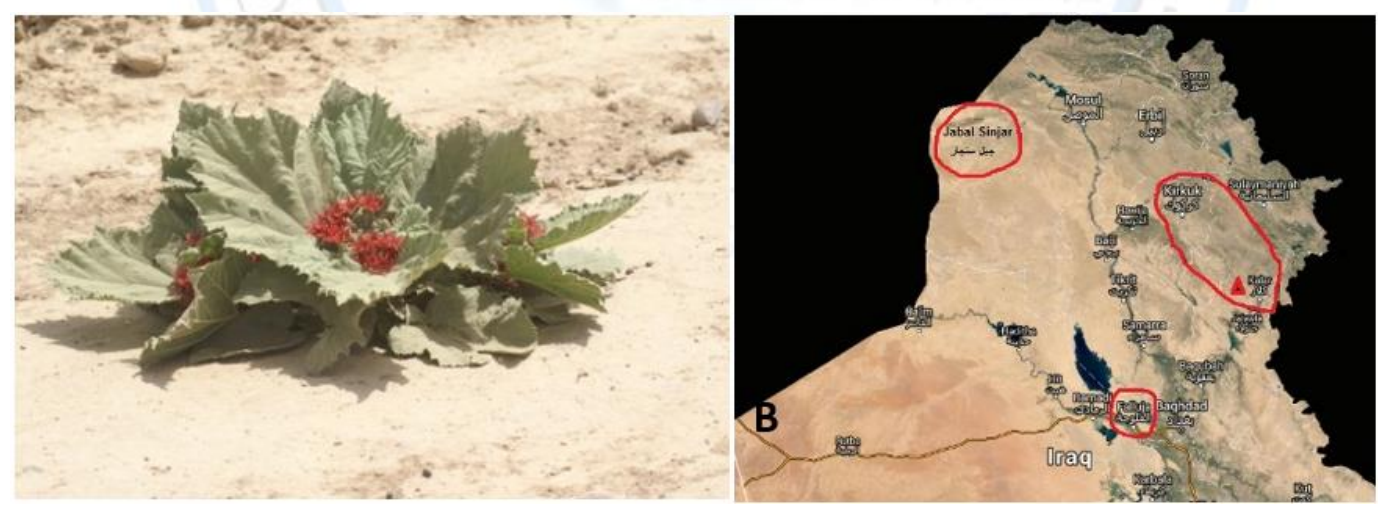

Plate 1: Species status of Glossostemon bruguier: A: G. bruguier in field, B: Geographic distribution of G. bruguier as last references, $\Delta$ site of collection.

The anatomical characters of the different parts of Glossostemon bruguieri have no references, our investigation showed that (root, stem, inflorescences and leaf petiole) rich with Schizogenous secretory ducts of mucilaginous materials with differentiation in density 


\section{Anatomical and Phytochemical Study of Glossostemon bruguieri}

(Desf.) Sterculiaceae in Kurdistan Region of Iraq

Khalid Faiq Darweesh
Karzan Mahmood Ahmed

and activity of the ducts which showed high ratio in stem (Plate 2, A) in both pith and cortex, also the cross section of stem showing irregular angular complete vascular cylinder and scattered secretory internal ducts in cortex (the smallest) and in pith (the largest) (Plate 2, E), and the cross section of inflorescence as extension to stem showed smallest duct cavities (Plate 2,B), while the cross section of root showed a large ducts only in cortex outer the vascular bundles (Plate 2,C) which characterized by surrounding cells with oil droplets(Plate 2,D), the leaf mesophyll is isobilateral and have cells with Crystalline spherical P-protein bodies (Plate 2,L) according to (Beck, 2010) which occur clearly dark spheres outside vascular bundles in both leaf and leaf petiole (Plate 2, G), no ducts in leaf except in the cortex of leaf petiole (Plate 2, F and $\mathrm{H}$ ), we suggest that ducts and its mucilaginous materials is the main source of phytochemicals in Moghat Glossostemon bruguieri in addition to oil droplets in roots and P-protein bodies of leaf and leaf petiole.The Indumentum study showed that all shoot parts covered by stellate trichome (Plate2, K) but the epidermis of stem and inflorescences contain special dendroid trichome (Plate2,I) consist of multicellular stalk and tree like branch in the top of stalk. Also the present of specialized glandular trichome (Plate 2, J) with multicellular stalk and multicellular glandular trichome this described by (Evert, 2006) and may be another source of some phytochemicals. 


\section{DIYALA JOURNAL FOR PURE SCIENCES}

Anatomical and Phytochemical Study of Glossostemon bruguieri

(Desf.) Sterculiaceae in Kurdistan Region of Iraq

Khalid Faiq Darweesh
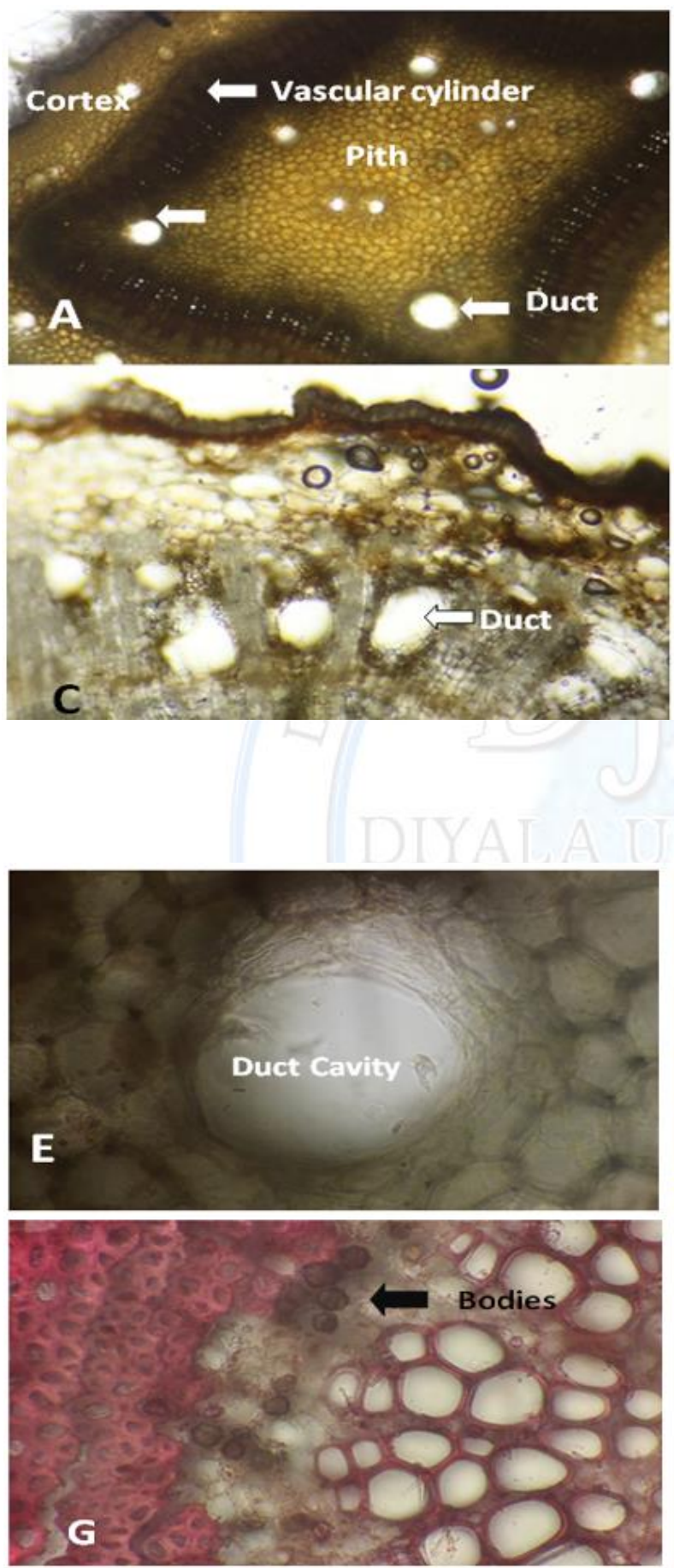

Karzan Mahmood Ahmed
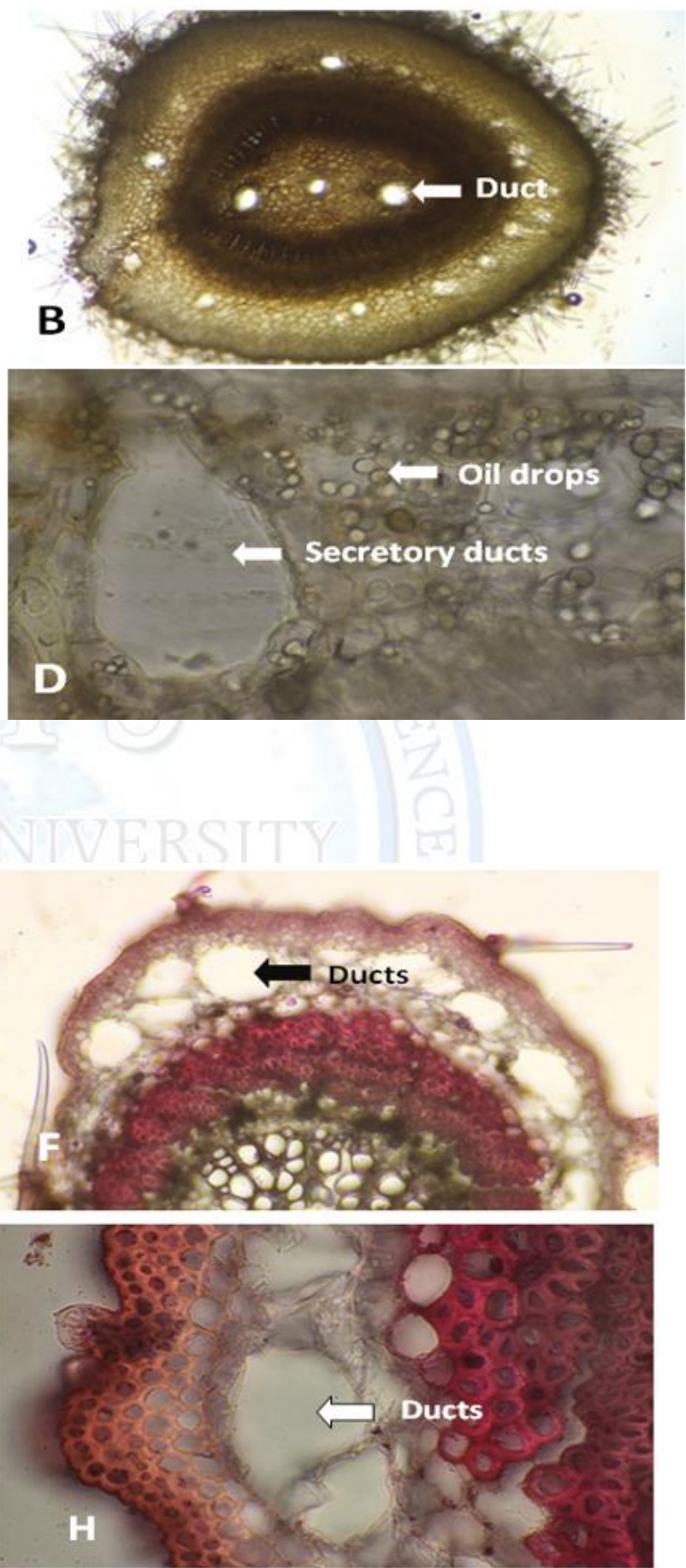

Followed 


\section{DIVALA JOURNAL FOR PURE SCIENCES}

Anatomical and Phytochemical Study of Glossostemon bruguieri

(Desf.) Sterculiaceae in Kurdistan Region of Iraq
Khalid Faiq Darweesh
Karzan Mahmood Ahmed
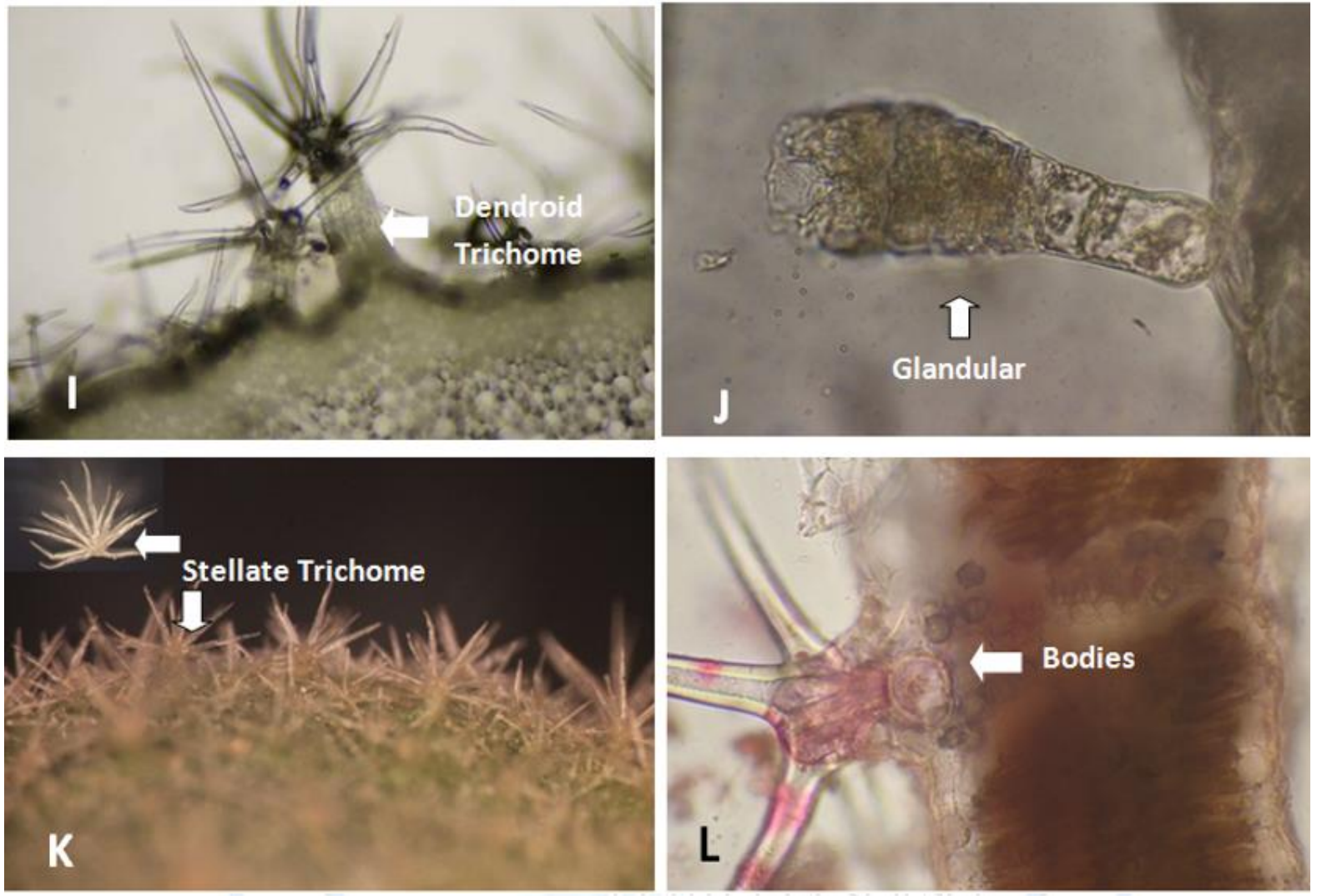

Plate 2: Anatomical characters of Glossostemon bruguieri: A: The cross section of stem, B: The cross section of inflorescence, C: The cross section of root, D: Enlargement part of the cross section of root, E: Enlargement section showing the duct cavity F: The cross section of leaf petiole, G; Enlargement part of section of leaf petiole showing bodies, $\mathrm{H}$ : Enlargement part of section of leaf petiole showing large duct cavity, I: Dendroid trichome, J: Specialized glandular trichome, K: Stellate trichome and L: Stellate trichome with secretory cells and some bodies in the basal cells of trichome. 
Anatomical and Phytochemical Study of Glossostemon bruguieri

(Desf.) Sterculiaceae in Kurdistan Region of Iraq

Khalid Faiq Darweesh

Karzan Mahmood Ahmed

\section{Phytochemical compounds in G. bruguieri leaf, stem, flower and inflorescence}

Methanolic extraction was obtained by maceration extraction from the leaf, flower, stem and inflorescence of Glossostemon bruguieri with yields of $7.28 \%, 3.6 \%, 8.4 \%$ and $4.41 \%$ respectively. The results are obtained by Gas Chromatography and Mass Spectroscopy analyses of methanol extraction of the G. bruguieri (Table 1 - 4). This table showed that 50 compounds (80.82\%) of flower, 43 compounds (71.09\%) of inflorescence, 39 compounds $(72.14 \%)$ of leaf and 55 compounds $(84.76 \%)$ of stem were identified.

The number of hydrocarbon compounds in inflorescence was higher compared to other three plant parts according for $24.96 \%$ of the total amount of volatile compounds, but approximately the similar number of hydrocarbon compounds was presence in flower (10 compounds, $14.73 \%$ ), leaf (10 compounds, 14.91\%) and stem (8 compounds, $7.22 \%$ ). Stem parts of G. bruguieri showed contain higher number of nitrogen compounds was 7 (2.89\%) and lower number presence in inflorescence was $1(0.69 \%)$, beside stem and inflorescence the number of nitrogen compounds was 3 and 5 in both leaf and flower but the proportion increased from $1.32 \%$ in leaf to $6.21 \%$ in flower. On the other hand the number of ester compounds was 3 in inflorescence and leaf but also the yield of volatile compounds deeply similar $5.17 \%$ in inflorescence to $5.21 \%$ in leaf, while 5 compounds in flower and 2 in stem with percentage ratio $9.19 \%$ and $1.98 \%$ respectively. The higher number of acid compounds was found in stem (12 compounds with ratio 10.89\%) and lower number (7 compounds with ratio 6.46\%) in flower while 8 and 9 compounds was presence in both inflorescence and leave with relative contribute $(8.77 \%$ and $8.96 \%)$ respectively. Finally the relative proportion of alcohols compounds in flower $29.49 \%$ and leave $28.04 \%$ increased as compound to those in stem $25.01 \%$ and inflorescence $20.28 \%$, and in contrast apparent in the percentage ratio of aldehydes and ketones compounds which was $12.69 \%$ in stem, $3.45 \%$ in flower, $3.36 \%$ in inflorescence and $2.62 \%$ in leaf. The significant differences in the chemical constituents founds in these four parts provide compelling evidence that leaves, stems, flowers and inflorescences must be used in the treatment of various disease. Of the various classes of compound identified, alcohols and hydrocarbons constituted the major part in inflorescence, leaf, flower and stem. Cyclohexanol was the most abundant hydrocarbon compound in all 
Anatomical and Phytochemical Study of Glossostemon bruguieri

(Desf.) Sterculiaceae in Kurdistan Region of Iraq

Khalid Faiq Darweesh
Karzan Mahmood Ahmed

plant parts with relative proportion $16.97 \%$ in flower, $15.04 \%$ in inflorescence, $16.72 \%$ in leaf and $14.87 \%$ in stem. Beside this compound, other major volatile compounds found in flower were, gamma.-sitosterol (6.76\%), hexatriacontane (4.19\%), 1-(+)-Ascorbic acid 2,6dihexadecanoate $(2.80 \%)$, Tetradecyl trifluoroacetate $(2.61 \%)$, theobromine $(2.48 \%)$, 1 Tetradecene (2.38\%), n-Nonadecanol-1 (2.32\%), 2,2-Dimethoxybutane (2.31\%), N-MethoxyNmethylacetamide (2.23\%), Sucrose $(2.13 \%)$ and n-Propyl acetate (2.01\%). Also the predominant chemical constituents found in inflorescence are as 2,2-dimethoxybutane (3.70\%), Tetradecyl trifluoroacetate (3.53\%), hexanal (2.75\%), 1,2,4-Benzenetricarboxylic acid, 1,2-dimethyl ester (2.70\%), hexatriacontane (2.57\%) 1-Tetradecene (2.48\%), 1,4Benzenediol, 2,5-bis(1,1-dimethylethyl)- (2.26\%) and 9-Tricosene (Z)- (2.08\%). Similary the leading chemical compounds were obtained in leaf are as 2,2-dimethoxybutane $(5.35 \%)$, campesterol (5.14\%), n-tridecyl ester (3.31\%), 1-Tetradecene (2.83\%), Dodecane (2.45\%),Trifluoroacetic acid, 1,3,5-Cycloheptatriene, 6-methyl-1-(-6-methyl-1,3,5cycloheptatrien-1-yl-)- (2.41\%) and Nonadecanol-1 (2.24\%).

Finally some high percentage chemical compounds were identified in stem included; Sucrose (16.94\%), 3-Deoxy-d-mannoic lactone (4.94\%), 3-Deoxy-d-mannoic acid (4.07\%), Propanal, 2,3-dihydroxy- (3.78\%), Glycerin (3.33\%), Stegmasterol (2.78\%), 1-Tridecene (2.36\%), Cholesterol $(2.42 \%)$. In the earlier study, it has been reported that the identified compounds exert significant biological activity for example Gamma-Sitosterol has antioxidant, antibacterial and prophylactic activities (Venkata, R, et al., 2012). Eicosane has a good activity against fungal, bacterial, tumor and cytotoxic effects (Hsouna, A.B, et al., 2011). Hexadecanoic acid, methyl ester displaying antifungal, antioxidant, hypocholesterolemic nematicide, pesticide, anti-androgenic flavour, haemolytic, 5-Alpha reductase inhibitor, potent antimicrobial activity (Hema et al., 2011). Dibutyl phthalate is used as antifungal, antimicrobial agent and antimalarial (Elija, et al., 2012). Dodecane enhances antifungal activity (Cheila, et al., 2012). Thymine could be used a target for actions of 5-fluorouracil (5FU) in cancer treatment (Hofreiter, et al., 2001). Lupeol has a complex pharmacology, displaying antiprotozal, antimicrobial, anti-inflammatory, antitumor and chemoprevention 
Anatomical and Phytochemical Study of Glossostemon bruguieri

(Desf.) Sterculiaceae in Kurdistan Region of Iraq

Khalid Faiq Darweesh
Karzan Mahmood Ahmed

properties (Margareth and Miranda, 2009). Theobromine is used as a vasodilator (a blood vessel widener), a diuretic and heart stimulant (William, 1943).

Table 1: GC-MS identified components of the Glossostemon bruguieri flower extract

\begin{tabular}{|c|c|c|c|c|c|}
\hline No. & $\begin{array}{c}\text { R.Ti } \\
\text { me }\end{array}$ & $\begin{array}{l}\text { M. } \\
\text { Wt }\end{array}$ & $\begin{array}{l}\text { Molecular } \\
\text { formula }\end{array}$ & $\begin{array}{c}\text { Flower } \\
\%\end{array}$ & Name and Class of Compound \\
\hline & & & & & Hydrocarbon Compounds \\
\hline 1 & 3.45 & 106 & $\mathrm{C} 8 \mathrm{H} 10$ & 0.73 & Benzene, 1,3-dimethyl- \\
\hline 2 & 4.67 & 156 & $\mathrm{C} 11 \mathrm{H} 24$ & 1.19 & Undecane \\
\hline 3 & 4.99 & 182 & $\mathrm{C} 13 \mathrm{H} 26$ & 1.68 & 1-Tridecene \\
\hline 4 & 6.34 & 210 & C16H18 & 1.10 & 1,1'-Biphenyl, 2,2',5,5'-tetramethyl- \\
\hline 5 & 5.56 & 196 & $\mathrm{C} 14 \mathrm{H} 28$ & 2.38 & 1-Tetradecene \\
\hline 6 & 7.45 & 282 & $\mathrm{C} 20 \mathrm{H} 42$ & 0.46 & Eicosane \\
\hline 7 & 4.22 & 170 & $\mathrm{C} 12 \mathrm{H} 26$ & 1.39 & Octane, 3,4,5,6-tetramethyl- \\
\hline 8 & 8.38 & 506 & $\mathrm{C} 36 \mathrm{H} 74$ & 4.19 & Hexatriacontane \\
\hline 9 & 8.79 & 478 & $\mathrm{C} 34 \mathrm{H} 70$ & 0.70 & Tetratriacontane \\
\hline \multirow[t]{2}{*}{10} & 10.14 & 410 & $\mathrm{C} 30 \mathrm{H} 50$ & 0.91 & $\begin{array}{l}2,6,10,14,18,22-\text { Tetracosahexaene, } 2,6,10,15,19,23- \\
\text { hexamethyl-, }\end{array}$ \\
\hline & & & & & Nitrogen Copmounds \\
\hline 11 & 2.58 & 103 & $\mathrm{C} 4 \mathrm{H} 9 \mathrm{NO} 2$ & 2.23 & N-Methoxy-N-methylacetamide \\
\hline 12 & 4.81 & 102 & $\mathrm{C} 4 \mathrm{H} 10 \mathrm{~N} 2 \mathrm{O}$ & 0.39 & 2-Propanamine, N-methyl-N-nitroso- \\
\hline 13 & 4.94 & 115 & $\mathrm{C} 5 \mathrm{H} 9 \mathrm{NO} 2$ & 0.46 & 5-Methoxypyrrolidin-2-one \\
\hline 14 & 6.75 & 194 & $\mathrm{C} 8 \mathrm{H} 10 \mathrm{~N} 4 \mathrm{O} 2$ & 0.65 & Caffeine \\
\hline \multirow[t]{2}{*}{15} & 6.79 & 180 & C7H8N4O2 & 2.48 & Theobromine \\
\hline & & & 78 & & Ester Compounds \\
\hline 16 & 2.80 & 102 & $\mathrm{C} 5 \mathrm{H} 10 \mathrm{O} 2$ & 2.01 & n-Propyl acetate \\
\hline 17 & 2.91 & 118 & $\mathrm{C} 5 \mathrm{H} 10 \mathrm{O} 3$ & 0.54 & 1,2-Propanediol, 2-acetate \\
\hline 18 & 6.04 & 310 & $\mathrm{C} 16 \mathrm{H} 29 \mathrm{~F} 3 \mathrm{O} 2$ & 2.61 & Tetradecyl trifluoroacetate \\
\hline 19 & 6.87 & 652 & C38H68O8 & 2.80 & 1-(+)-Ascorbic acid 2,6-dihexadecanoate \\
\hline \multirow[t]{2}{*}{20} & 6.94 & 254 & $\mathrm{C} 1630 \mathrm{O} 2$ & 1.23 & 13-Tetradecen-1-ol acetate \\
\hline & & & & & Acid Compounds \\
\hline 21 & 2.71 & 128 & $\mathrm{C} 6 \mathrm{H} 8 \mathrm{O} 3$ & 1.40 & 2-Propenoic acid, oxiranylmethyl ester \\
\hline 22 & 5.52 & 216 & $\mathrm{C} 12 \mathrm{H} 24 \mathrm{O} 3$ & 0.57 & $\begin{array}{l}\text { Propanoic acid, 2-methyl-, 2,2-dimethyl-1-(2-hydroxy-1- } \\
\text { methylethyl)propyl ester }\end{array}$ \\
\hline 23 & 5.95 & 258 & $\mathrm{C} 14 \mathrm{H} 26 \mathrm{O} 4$ & 0.30 & Hexanedioic acid, mono(2-ethylhexyl)ester \\
\hline 24 & 6.09 & 286 & $\mathrm{C} 16 \mathrm{H} 30 \mathrm{O} 4$ & 0.98 & $\begin{array}{l}\text { Propanoic acid, 2-methyl-, 1-(1,1-dimethylethyl)-2- } \\
\text { methyl-1,3-propanediyl ester }\end{array}$ \\
\hline 25 & 6.71 & 278 & $\mathrm{C} 16 \mathrm{H} 22 \mathrm{O} 4$ & 0.74 & 1,2-Benzenedicarboxylic acid, bis(2-methylpropyl) ester \\
\hline 26 & 7.22 & 322 & $\mathrm{C} 21 \mathrm{H} 38 \mathrm{O} 2$ & 0.97 & 10,13-Eicosadienoic acid, methyl ester \\
\hline \multirow[t]{2}{*}{27} & 8.66 & 278 & $\mathrm{C} 16 \mathrm{H} 22 \mathrm{O} 4$ & 1.50 & 1,2-Benzenedicarboxylic acid, mono(2-ethylhexyl) ester \\
\hline & & & & & Alcohol Compounds \\
\hline 28 & 3.62 & 100 & $\mathrm{C} 6 \mathrm{H} 12 \mathrm{O}$ & 16.96 & Cyclohexanol \\
\hline 29 & 4.38 & 130 & $\mathrm{C} 8 \mathrm{H} 18 \mathrm{O}$ & 0.30 & 2-Propyl-1-pentanol \\
\hline 30 & 5.15 & 134 & $\mathrm{C} 5 \mathrm{H} 10 \mathrm{O} 4$ & 0.59 & 1,2,3-Propanetriol, 1-acetate \\
\hline
\end{tabular}


Anatomical and Phytochemical Study of Glossostemon bruguieri

(Desf.) Sterculiaceae in Kurdistan Region of Iraq

Khalid Faiq Darweesh

Karzan Mahmood Ahmed

\begin{tabular}{|l|c|c|c|c|c|}
\hline $\mathbf{3 1}$ & 6.29 & 238 & $\mathrm{C} 9 \mathrm{H} 18 \mathrm{O} 7$ & 1.08 & 6-O-Methyl-2,4-methylene-.beta.-sedoheptitol \\
\hline $\mathbf{3 2}$ & 6.44 & 414 & $\mathrm{C} 29 \mathrm{H} 50 \mathrm{O}$ & 6.76 & gamma.-Sitosterol \\
\hline $\mathbf{3 3}$ & 6.49 & 284 & $\mathrm{C} 19 \mathrm{H} 40 \mathrm{O}$ & 2.32 & n-Nonadecanol-1 \\
\hline $\mathbf{3 4}$ & 7.02 & 238 & $\mathrm{C} 15 \mathrm{H} 26 \mathrm{O} 2$ & 0.31 & Cedrane-8,13-diol \\
\hline $\mathbf{3 5}$ & 8.25 & 426 & $\mathrm{C} 30 \mathrm{H} 50 \mathrm{O}$ & 1.17 & Lupeol \\
\hline & & & & & Aldehyde and ketone Compounds \\
\hline $\mathbf{3 6}$ & 4.87 & 144 & $\mathrm{C} 6 \mathrm{H} 8 \mathrm{O} 4$ & 0.59 & 4H-Pyran-4-one, 2,3-dihydro-3,5-dihydroxy-6-methyl- \\
\hline $\mathbf{3 7}$ & 6.62 & 268 & $\mathrm{C} 18 \mathrm{H} 36 \mathrm{O}$ & 1.47 & 2-Pentadecanone, 6,10,14-trimethyl- \\
\hline $\mathbf{3 8}$ & 7.31 & 238 & $\mathrm{C} 16 \mathrm{H} 30 \mathrm{O}$ & 2.39 & cis-9-Hexadecenal \\
\hline
\end{tabular}

\begin{tabular}{|l|l|l|l|l|c|}
\hline & & & & & Other Compounds \\
\hline $\mathbf{3 9}$ & 2.52 & 118 & C7H15F & 1.18 & Heptane, 1-fluoro- \\
\hline $\mathbf{4 0}$ & 2.66 & 118 & C6H14O2 & 2.31 & 2,2-Dimethoxybutane \\
\hline $\mathbf{4 1}$ & 3.53 & 136 & C9H12O & 0.49 & Benzene, (2 methoxyethyl)- \\
\hline $\mathbf{4 2}$ & 3.55 & 166 & C10H14O2 & 0.35 & 4-(methoxymethyl)- 2,6-dimethyl-phenol \\
\hline $\mathbf{4 3}$ & 4.06 & 106 & C4H10O3 & 0.67 & Tripropylene glycol monomethyl ether \\
\hline $\mathbf{4 4}$ & 4.15 & 144 & C6H8O2 & 0.31 & 2,4-Dihydroxy-2,5-dimethyl-3(2H)-furan-3-one \\
\hline $\mathbf{4 5}$ & 4.17 & 102 & C4H6O3 & 0.49 & 2-Hydroxy-gamma-butyrolactone \\
\hline $\mathbf{4 6}$ & 4.59 & 126 & C7H10O2 & 0.44 & Cyclopentane, 1-acetyl-1,2-epoxy- \\
\hline $\mathbf{4 7}$ & 5.19 & 210 & C7H14O7 & 0.94 & Heptose \\
\hline $\mathbf{4 8}$ & 5.71 & 342 & C12H22O11 & 2.13 & Sucrose \\
\hline $\mathbf{4 9}$ & 7.99 & 324 & C21H40O2 & 0.55 & 4,8,12,16-Tetramethylheptadecan-4-olide \\
\hline $\mathbf{5 0}$ & 5.09 & 120 & C8H8O & 0.52 & Benzofuran, 2,3-dihydro- \\
\hline \multicolumn{7}{|c|}{ Total identified compounds } & $\mathbf{8 0 . 8 2 \%}$ & \\
\hline
\end{tabular}

Table 2: GC-MS identified components of the Glossostemon bruguieri inflorescence extract.

\begin{tabular}{|c|c|c|c|c|c|}
\hline No. & $\begin{array}{c}\text { R.Ti } \\
\text { me }\end{array}$ & $\begin{array}{c}\text { M. } \\
\text { Wt }\end{array}$ & $\begin{array}{c}\text { Molecular } \\
\text { formula }\end{array}$ & $\begin{array}{c}\text { Infloresce } \\
\text { nce\% }\end{array}$ & Name and Class of Compound \\
\hline & & & & & Hydrocarbon Compounds \\
\hline $\mathbf{1}$ & 5.11 & 168 & $\mathrm{C} 12 \mathrm{H} 24$ & 0.52 & Cyclopropane, 1-methyl-2-octyl- \\
\hline $\mathbf{2}$ & 5.19 & 156 & $\mathrm{C} 11 \mathrm{H} 24$ & 1.83 & Undecane \\
\hline $\mathbf{3}$ & 6.31 & 168 & $\mathrm{C} 12 \mathrm{H} 24$ & 1.60 & Cyclopropane, nonyl- \\
\hline $\mathbf{4}$ & 7.89 & 196 & $\mathrm{C} 14 \mathrm{H} 28$ & 2.48 & 1-Tetradecene \\
\hline $\mathbf{5}$ & 4.60 & 170 & $\mathrm{C} 12 \mathrm{H} 26$ & 1.04 & Dodecane \\
\hline $\mathbf{6}$ & 8.02 & 252 & $\mathrm{C} 18 \mathrm{H} 36$ & 1.96 & Cyclodecane, methyl- \\
\hline $\mathbf{7}$ & 9.02 & 154 & $\mathrm{C} 11 \mathrm{H} 22$ & 0.37 & Benzene, (3,3-dimethyldecyl)- \\
\hline $\mathbf{8}$ & 9.41 & 246 & $\mathrm{C} 18 \mathrm{H} 30$ & 0.52 & Benzene, (1-ethylnonyl)- \\
\hline $\mathbf{9}$ & 9.59 & 232 & $\mathrm{C} 17 \mathrm{H} 28$ & 0.44 & Benzene, (1-methyldecyl)- \\
\hline $\mathbf{1 0}$ & 9.78 & 232 & $\mathrm{C} 17 \mathrm{H} 28$ & 0.37 & 1,1'-Biphenyl, 2,2',5,5'-tetramethyl- \\
\hline $\mathbf{1 1}$ & 9.87 & 210 & $\mathrm{C} 16 \mathrm{H} 18$ & 0.65 & Benzene, (2,3-dimethyldecyl)- \\
\hline $\mathbf{1 2}$ & 9.91 & 246 & $\mathrm{C} 18 \mathrm{H} 30$ & 0.34 & \\
\hline
\end{tabular}


Anatomical and Phytochemical Study of Glossostemon bruguieri

(Desf.) Sterculiaceae in Kurdistan Region of Iraq

Khalid Faiq Darweesh

Karzan Mahmood Ahmed

\begin{tabular}{|c|c|c|c|c|c|}
\hline $\mathbf{1 3}$ & 9.96 & 238 & $\mathrm{C} 17 \mathrm{H} 34$ & 0.53 & Cyclopropane, 1-methyl-1-(2-methylpropyl)-2-nonyl- \\
\hline $\mathbf{1 4}$ & 10.05 & 182 & $\mathrm{C} 13 \mathrm{H} 26$ & 0.32 & Cyclohexane, 2-butyl-1,1,3-trimethyl- \\
\hline $\mathbf{1 5}$ & 10.15 & 322 & $\mathrm{C} 23 \mathrm{H} 46$ & 2.08 & 9-Tricosene, (Z)- \\
\hline $\mathbf{1 6}$ & 11.14 & 410 & $\mathrm{C} 30 \mathrm{H} 50$ & 6.97 & $\begin{array}{c}2,6,10,14,18,22-\text { Tetracosahexaene, 2,6,10,15,19,23- } \\
\text { hexamethyl-, (all-E)- }\end{array}$ \\
\hline $\mathbf{1 7}$ & 12.76 & 478 & $\mathrm{C} 34 \mathrm{H} 70$ & 0.37 & Tetratriacontane \\
\hline $\mathbf{1 8}$ & 13.23 & 506 & $\mathrm{C} 36 \mathrm{H} 74$ & 2.57 & Hexatriacontane \\
\hline & & & & & Nitrogen Compounds \\
\hline $\mathbf{1 9}$ & 9.74 & 281 & $\mathrm{C} 17 \mathrm{H} 15 \mathrm{NO} 3$ & 0.69 & Acetamide, N-(acetyloxy)-N-9H-fluoren-2-yl- \\
\hline & & & & & Ester Compounds \\
\hline $\mathbf{2 0}$ & 2.66 & 102 & $\mathrm{C} 5 \mathrm{H} 10 \mathrm{O} 2$ & 0.69 & n-Propyl acetate \\
\hline $\mathbf{2 1}$ & 9.13 & 310 & $\mathrm{C} 16 \mathrm{H} 29 \mathrm{~F} 3 \mathrm{O} 2$ & 3.53 & Tetradecyl trifluoroacetate \\
\hline $\mathbf{2 2}$ & 10.96 & 278 & $\mathrm{C} 16 \mathrm{H} 22 \mathrm{O} 4$ & 0.95 & Dibutyl phthalate
\end{tabular}

\begin{tabular}{|c|c|c|c|c|c|}
\hline & & & 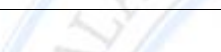 & & Acid Compounds \\
\hline 23 & 7.98 & 216 & $\mathrm{C} 12 \mathrm{H} 24 \mathrm{O} 3$ & 0.47 & $\begin{array}{l}\text { Propanoic acid, 2-methyl-, 3-hydroxy-2,4,4- } \\
\text { trimethylpentyl ester }\end{array}$ \\
\hline 24 & 9.23 & 286 & $\mathrm{C} 16 \mathrm{H} 30 \mathrm{O} 4$ & 0.59 & $\begin{array}{l}\text { Propanoic acid, 2-methyl-, 1-(1,1-dimethylethyl)-2- } \\
\text { methyl-1,3-propanediyl ester }\end{array}$ \\
\hline 25 & 10.55 & 278 & $\mathrm{C} 16 \mathrm{H} 22 \mathrm{O} 4$ & 0.29 & 1,2-Benzenedicarboxylic acid, bis(2-methylpropyl) ester \\
\hline 26 & 10.75 & 270 & $\mathrm{C} 17 \mathrm{H} 34 \mathrm{O} 2$ & 1.41 & Hexadecanoic acid, methyl ester \\
\hline 27 & 10.89 & 242 & $\mathrm{C} 15 \mathrm{H} 30 \mathrm{O} 2$ & 0.85 & Pentadecanoic acid \\
\hline 28 & 11.50 & 322 & $\mathrm{C} 21 \mathrm{H} 38 \mathrm{O} 2$ & 1.59 & cis-11,14-Eicosadienoic acid, methyl ester \\
\hline 29 & 11.59 & 298 & $\mathrm{C} 19 \mathrm{H} 38 \mathrm{O} 2$ & 0.87 & Heptadecanoic acid, 10-methyl-, methyl ester \\
\hline 30 & 14.50 & 238 & C11H10O6 & 2.70 & 1,2,4-Benzenetricarboxylic acid, 1,2-dimethyl ester \\
\hline & & & 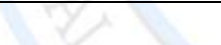 & & Alcohol Compounds \\
\hline 31 & 4.12 & 100 & $\mathrm{C} 6 \mathrm{H} 12 \mathrm{O}$ & 15.04 & Cyclohexanol \\
\hline 32 & 11.05 & 284 & $\mathrm{C} 19 \mathrm{H} 40 \mathrm{O}$ & 0.76 & n-Nonadecanol-1 \\
\hline 33 & 11.44 & 355 & $\mathrm{C} 24 \mathrm{H} 50 \mathrm{O}$ & 0.29 & $\mathrm{n}$-Tetracosanol-1 \\
\hline 34 & 11.65 & 268 & $\mathrm{C} 18 \mathrm{H} 36 \mathrm{O}$ & 1.28 & Oleyl Alcohol \\
\hline 35 & 14.55 & 222 & $\mathrm{C} 14 \mathrm{H} 22 \mathrm{O} 2$ & 2.26 & 1,4-Benzenediol, 2,5-bis(1,1-dimethylethyl)- \\
\hline 36 & 15.04 & 222 & $\mathrm{C} 14 \mathrm{H} 22 \mathrm{O} 2$ & 0.65 & 4,6-di-tert-Butylresorcinol \\
\hline & & & & & Aldehyde and ketone Compounds \\
\hline 37 & 2.52 & 100 & $\mathrm{C} 6 \mathrm{H} 12 \mathrm{O}$ & 2.75 & Hexanal \\
\hline 38 & 10.38 & 226 & $\mathrm{C} 15 \mathrm{H} 30 \mathrm{O}$ & 0.61 & Pentadecanal- \\
\hline & & & & & Other Compounds \\
\hline 39 & 2.78 & 118 & $\mathrm{C} 6 \mathrm{H} 12 \mathrm{O} 2$ & 3.70 & 2,2-Dimethoxybutane \\
\hline 40 & 3.88 & 204 & $\mathrm{C} 12 \mathrm{H} 25 \mathrm{Cl}$ & 1.45 & Dodecane, 1-chloro- \\
\hline 41 & 8.22 & 342 & $\mathrm{C} 12 \mathrm{H} 22 \mathrm{O} 11$ & 0.51 & Sucrose \\
\hline 42 & 11.87 & 914 & $\mathrm{C} 54 \mathrm{H} 108 \mathrm{Br} 2$ & 1.93 & Tetrapentacontane, 1,54-dibromo- \\
\hline 43 & 12.65 & 350 & $\mathrm{C} 15 \mathrm{H} 24 \mathrm{~F} 6 \mathrm{O} 2$ & 0.27 & $\begin{array}{c}\text { 1,3-Dioxolane, 4-ethyl-5-octyl-2,2-bis(trifluoromethyl)-, } \\
\text { cis- }\end{array}$ \\
\hline \multicolumn{4}{|c|}{ Total identified compounds } & $71.09 \%$ & \\
\hline
\end{tabular}


Anatomical and Phytochemical Study of Glossostemon bruguieri

(Desf.) Sterculiaceae in Kurdistan Region of Iraq

Khalid Faiq Darweesh

Karzan Mahmood Ahmed

Table 3: GC-MS identified components of the Glossostemon bruguieri leaf extract.

\begin{tabular}{|c|c|c|c|c|c|}
\hline No. & $\begin{array}{l}\text { R.Ti } \\
\text { me }\end{array}$ & $\begin{array}{l}\text { M. } \\
\text { Wt }\end{array}$ & $\begin{array}{l}\text { Molecular } \\
\text { formula }\end{array}$ & Leaf \% & Name and Class of Compound \\
\hline & & & & & Hydrocarbon Compounds \\
\hline 1 & 3.46 & 106 & $\mathrm{C} 8 \mathrm{H} 10$ & 1.16 & Benzene, 1,3-dimethyl- \\
\hline 2 & 3.14 & 140 & $\mathrm{C} 10 \mathrm{H} 20$ & 0.46 & 1-Decene \\
\hline 3 & 3.23 & 156 & $\mathrm{C} 11 \mathrm{H} 24$ & 0.62 & Undecane \\
\hline 4 & 4.20 & 170 & $\mathrm{C} 12 \mathrm{H} 26$ & 2.45 & Dodecane \\
\hline 5 & 4.76 & 182 & $\mathrm{C} 13 \mathrm{H} 26$ & 1.91 & 1-Tridecene \\
\hline 6 & 5.57 & 196 & $\mathrm{C} 14 \mathrm{H} 28$ & 2.83 & 1-Tetradecene \\
\hline 7 & 6.00 & 182 & $\mathrm{C} 13 \mathrm{H} 26$ & 0.50 & Cyclohexane, 2-butyl-1,1,3-trimethyl- \\
\hline 8 & 6.37 & 210 & $\mathrm{C} 16 \mathrm{H} 18$ & 2.41 & $\begin{array}{l}\text { 1,3,5-Cycloheptatriene, 6-methyl-1-(6-methyl-1,3,5- } \\
\text { cycloheptatrien-1-yl)- }\end{array}$ \\
\hline 9 & 6.43 & 210 & $\mathrm{C} 16 \mathrm{H} 18$ & 1.50 & 1,1'-Biphenyl, 2,2',5,5'-tetramethyl- \\
\hline \multirow[t]{2}{*}{10} & 6.48 & 252 & $\mathrm{C} 18 \mathrm{H} 36$ & 1.07 & 8-Heptadecene, 8-methyl-, (E)- \\
\hline & & & $=$ & & Nitrogen Compounds \\
\hline 11 & 4.45 & 254 & $\mathrm{C} 16 \mathrm{H} 18 \mathrm{~N} 2 \mathrm{O}$ & 0.20 & Pyrido[2,3-g]indole, 5-methoxy-2,3,7,9-tetramethyl- \\
\hline 12 & 8.38 & 355 & $\mathrm{C} 21 \mathrm{H} 25 \mathrm{NO} 4$ & 0.84 & $\begin{array}{l}\text { 1H-Indole-2-carboxylic acid, 6-(4-ethoxyphenyl)-3- } \\
\text { methyl-4-oxo-4,5,6,7-tetrahydro-, isopropyl ester }\end{array}$ \\
\hline \multirow[t]{2}{*}{13} & 10.35 & 402 & $\mathrm{C} 23 \mathrm{H} 34 \mathrm{~N} 2 \mathrm{O} 4$ & 0.28 & $\begin{array}{c}\text { 5,5'-Di(ethoxycarbonyl)-3,3'-dimethyl-4,4'-dipropyl-2,2'- } \\
\text { dipyrrylmethane }\end{array}$ \\
\hline & & & & & Ester Compounds \\
\hline 14 & 6.97 & 278 & $\mathrm{C} 16 \mathrm{H} 22 \mathrm{O} 4$ & 1.74 & Dibutyl phthalate \\
\hline 15 & 6.99 & 254 & $\mathrm{C} 16 \mathrm{H} 30 \mathrm{O} 2$ & 1.72 & 13-Tetradecen-1-ol acetate \\
\hline \multirow{2}{*}{16} & 7.49 & 430 & $\mathrm{C} 22 \mathrm{H} 39 \mathrm{~F} 5 \mathrm{O} 2$ & 1.75 & Nonadecyl pentafluoropropionate \\
\hline & & & & & Acid Compounds \\
\hline 17 & 5.46 & 200 & $\mathrm{C} 12 \mathrm{H} 24 \mathrm{O} 2$ & 0.27 & Propanoic acid, nonyl ester \\
\hline 18 & 6.06 & 296 & $\mathrm{C} 15 \mathrm{H} 27 \mathrm{~F} 3 \mathrm{O} 2$ & 3.31 & Trifluoroacetic acid,n-tridecyl ester \\
\hline 19 & 6.11 & 286 & $\mathrm{C} 16 \mathrm{H} 30 \mathrm{O} 4$ & 1.52 & $\begin{array}{l}\text { Propanoic acid, 2-methyl-, 1-(1,1-dimethylethyl)-2- } \\
\text { methyl-1,3-propanediyl ester }\end{array}$ \\
\hline 20 & 6.75 & 374 & $\mathrm{C} 23 \mathrm{H} 34 \mathrm{O} 4$ & 0.98 & Phthalic acid, isobutyl undec-2-en-1-yl ester \\
\hline 21 & 6.83 & 270 & $\mathrm{C} 17 \mathrm{H} 34 \mathrm{O} 2$ & 1.72 & Hexadecanoic acid, methyl ester \\
\hline 22 & 6.91 & 404 & $\mathrm{C} 21 \mathrm{H} 41 \mathrm{BrO} 2$ & 0.17 & 6-Bromohexanoic acid, pentadecyl ester \\
\hline 23 & 6.79 & 316 & C15H16N4O4 & 0.30 & Pentadioic acid, dihydrazide, N2,N2'-bis(2-furfurylideno)- \\
\hline 24 & 7.80 & 296 & $\mathrm{C} 19 \mathrm{H} 36 \mathrm{O} 2$ & 0.46 & trans-13-Octadecenoic acid, methyl ester \\
\hline \multirow[t]{2}{*}{25} & 8.73 & 390 & $\mathrm{C} 24 \mathrm{H} 38 \mathrm{O} 4$ & 0.23 & 1,2-Benzenedicarboxylic acid, diisooctyl ester \\
\hline & & & & & Alcohol Compounds \\
\hline 26 & 3.58 & 130 & $\mathrm{C} 8 \mathrm{H} 18 \mathrm{O}$ & 0.24 & 1-Hexanol, 2-ethyl- \\
\hline 27 & 3.63 & 100 & $\mathrm{C} 6 \mathrm{H} 12 \mathrm{O}$ & 16.72 & Cyclohexanol \\
\hline 28 & 5.69 & 151 & C4H9NO5 & 0.42 & 1,3-Propanediol, 2-(hydroxymethyl)-2-nitro- \\
\hline 29 & 6.52 & 284 & $\mathrm{C} 19 \mathrm{H} 40 \mathrm{O}$ & 2.24 & n-Nonadecanol-1 \\
\hline 30 & 8.61 & 386 & $\mathrm{C} 27 \mathrm{H} 46 \mathrm{O}$ & 2.84 & Cholesterol \\
\hline 31 & 9.25 & 398 & $\mathrm{C} 28 \mathrm{H} 46 \mathrm{O}$ & 0.44 & Dihydrotachysterol \\
\hline \multirow[t]{2}{*}{32} & 10.21 & 400 & $\mathrm{C} 28 \mathrm{H} 48 \mathrm{O}$ & 5.14 & Campesterol \\
\hline & & & & & Aldehyde and ketone Compounds \\
\hline 33 & 4.57 & 182 & $\mathrm{C} 10 \mathrm{H} 11 \mathrm{ClO}$ & 0.24 & gamma.-Chlorobutyrophenone \\
\hline 34 & 5.73 & 162 & $\mathrm{C} 10 \mathrm{H} 10 \mathrm{O} 2$ & 0.51 & Ethanone, 1,1'-(1,3-phenylene)bis- \\
\hline
\end{tabular}


Anatomical and Phytochemical Study of Glossostemon bruguieri

(Desf.) Sterculiaceae in Kurdistan Region of Iraq

Khalid Faiq Darweesh

Karzan Mahmood Ahmed

\begin{tabular}{|c|c|c|c|c|c|}
\hline $\mathbf{3 5}$ & 7.26 & 238 & $\mathrm{C} 16 \mathrm{H} 30 \mathrm{O}$ & 1.87 & cis-9-Hexadecenal \\
\hline & & & & & Other Compounds \\
\hline $\mathbf{3 6}$ & 2.52 & 118 & $\mathrm{C} 7 \mathrm{H} 15 \mathrm{~F}$ & 1.95 & Heptane, 1-fluoro- \\
\hline $\mathbf{3 7}$ & 2.59 & 92 & $\mathrm{C} 3 \mathrm{H} 8 \mathrm{O} 3$ & 1.75 & Glycerin \\
\hline $\mathbf{3 8}$ & 2.66 & 118 & $\mathrm{C} 6 \mathrm{H} 14 \mathrm{O} 2$ & 5.35 & 2,2-Dimethoxybutane \\
\hline 39 & 4.32 & 144 & C8H16O2 & 1.98 & Cyclohexane, 1,1-dimethoxy- \\
\hline \multicolumn{3}{|c|}{ Total identified compounds } & $\mathbf{7 2 . 1 4 \%}$ & \\
\hline
\end{tabular}

Table 4: GC-MS identified components of the Glossostemon bruguieri stem extract.

\begin{tabular}{|c|c|c|c|c|c|}
\hline No. & $\begin{array}{c}\text { R. } \\
\text { Time }\end{array}$ & $\begin{array}{l}\text { M. } \\
\text { Wt }\end{array}$ & $\begin{array}{c}\text { Molecular } \\
\text { formula }\end{array}$ & Stem \% & Name and Class of Compound \\
\hline & & & & & Hydrocarbon Compounds \\
\hline 1 & 4.22 & 156 & $\mathrm{C} 11 \mathrm{H} 24$ & 0.64 & Octane, 2,3,3-trimethyl- \\
\hline 2 & 5.00 & 182 & $\mathrm{C} 13 \mathrm{H} 26$ & 2.36 & 1-Tridecene \\
\hline 3 & 4.68 & 156 & $\mathrm{C} 11 \mathrm{H} 24$ & 0.83 & Undecane \\
\hline 4 & 5.57 & 196 & $\mathrm{C} 14 \mathrm{H} 28$ & 1.45 & 1-Tetradecene \\
\hline 5 & 5.63 & 144 & $\mathrm{C} 11 \mathrm{H} 12$ & 0.18 & (1-Methylenebut-2-enyl)benzene \\
\hline 6 & 6.37 & 210 & C16H18 & 0.68 & 1,1'-Biphenyl, 2,2',5,5'-tetramethyl- \\
\hline 7 & 6.48 & 252 & $\mathrm{C} 18 \mathrm{H} 36$ & 0.81 & Cyclopropane, 1-(1,2-dimethylpropyl)-1-methyl-2-nonyl- \\
\hline \multirow[t]{2}{*}{8} & 10.21 & 410 & $\mathrm{C} 30 \mathrm{H} 50$ & 0.27 & $\begin{array}{l}2,6,10,14,18,22-\text { Tetracosahexaene, } 2,6,10,15,19,23- \\
\text { hexamethyl-, (all-E)- }\end{array}$ \\
\hline & & & & & Nitrogen Compounds \\
\hline 9 & 2.76 & 147 & $\mathrm{C} 9 \mathrm{H} 9 \mathrm{NO}$ & 0.65 & 3-(4-Hydroxyphenyl) propionitrile \\
\hline 10 & 3.22 & 116 & $\mathrm{C} 5 \mathrm{H} 12 \mathrm{~N} 2 \mathrm{O}$ & 0.28 & O-Butylisourea \\
\hline 11 & 3.45 & 149 & $\mathrm{C} 9 \mathrm{H} 11 \mathrm{NO}$ & 0.44 & Acetamide, N-(phenylmethyl)- \\
\hline 12 & 4.59 & 126 & $\mathrm{C} 5 \mathrm{H} 6 \mathrm{~N} 2 \mathrm{O} 2$ & 0.65 & Thymine \\
\hline 13 & 4.82 & 102 & $\mathrm{C} 4 \mathrm{H} 10 \mathrm{~N} 2 \mathrm{O}$ & 0.12 & 2-Propanamine, N-methyl-N-nitroso- \\
\hline 14 & 7.76 & 387 & $\mathrm{C} 24 \mathrm{H} 37 \mathrm{NO} 3$ & 0.65 & 3.beta.-Acetoxy-bisnor-5-cholenamide \\
\hline \multirow[t]{2}{*}{15} & 8.05 & 337 & $\mathrm{C} 22 \mathrm{H} 43 \mathrm{NO}$ & 0.10 & 13-Docosenamide, $(\mathrm{Z})$ - \\
\hline & & & & & Ester Compounds \\
\hline 16 & 6.06 & 310 & $\mathrm{C} 16 \mathrm{H} 29 \mathrm{~F} 3 \mathrm{O} 2$ & 1.03 & Tetradecyl trifluoroacetate \\
\hline \multirow[t]{2}{*}{17} & 6.97 & 278 & $\mathrm{C} 16 \mathrm{H} 22 \mathrm{O} 4$ & 0.91 & Dibutyl phthalate \\
\hline & & & & & Acid Compounds \\
\hline 18 & 2.92 & 102 & $\mathrm{C} 4 \mathrm{H} 6 \mathrm{O} 3$ & 0.59 & Propanoic acid, 2-oxo-, methyl ester \\
\hline 19 & 5.30 & 190 & $\mathrm{C} 8 \mathrm{H} 14 \mathrm{O} 5$ & 1.56 & $\begin{array}{c}\text { Propanoic acid, 3-(acetyloxy)-2-(hydroxymethyl)-, ethyl } \\
\text { ester, (+)- }\end{array}$ \\
\hline 20 & 5.46 & 158 & $\mathrm{C} 8 \mathrm{H} 14 \mathrm{O} 3$ & 0.69 & Butanoic acid, 3-oxo-, 2-methylpropyl ester \\
\hline 21 & 5.53 & 216 & $\mathrm{C} 12 \mathrm{H} 24 \mathrm{O} 3$ & 0.41 & $\begin{array}{l}\text { Propanoic acid, 2-methyl-, 2,2-dimethyl-1-(2-hydroxy-1- } \\
\text { methylethyl)propyl ester }\end{array}$ \\
\hline 22 & 6.02 & 316 & $\begin{array}{c}\mathrm{C} 13 \mathrm{H} 23 \mathrm{Cl} 3 \mathrm{O} \\
2\end{array}$ & 0.44 & Trichloroacetic acid, undecyl ester \\
\hline 23 & 6.19 & 180 & C6H12O6 & 4.07 & 3-Deoxy-d-mannonic acid \\
\hline 24 & 6.75 & 278 & $\mathrm{C} 16 \mathrm{H} 22 \mathrm{O} 4$ & 0.92 & 1,2-Benzenedicarboxylic acid, bis(2-methylpropyl) ester \\
\hline 25 & 6.83 & 270 & $\mathrm{C} 17 \mathrm{H} 34 \mathrm{O} 2$ & 0.46 & Hexadecanoic acid, methyl ester \\
\hline 26 & 6.91 & 652 & $\mathrm{C} 38 \mathrm{H} 68 \mathrm{O} 2$ & 0.93 & 1-(+)-Ascorbic acid 2,6-dihexadecanoate \\
\hline 27 & 7.36 & 280 & $\mathrm{C} 18 \mathrm{H} 32 \mathrm{O} 2$ & 0.44 & 9,12-Octadecadienoic acid (Z,Z)- \\
\hline 28 & 7.41 & 312 & $\mathrm{C} 2 \mathrm{OH} 40 \mathrm{O} 2$ & 0.12 & Eicosanoic acid \\
\hline
\end{tabular}




\section{Anatomical and Phytochemical Study of Glossostemon bruguieri}

(Desf.) Sterculiaceae in Kurdistan Region of Iraq

Khalid Faiq Darweesh
Karzan Mahmood Ahmed

\begin{tabular}{|c|c|c|c|c|c|}
\hline $\mathbf{2 9}$ & 8.73 & 278 & $\mathrm{C} 16 \mathrm{H} 22 \mathrm{O} 4$ & 0.26 & 1,2-Benzenedicarboxylic acid, mono(2-ethylhexyl) ester \\
\hline $\mathbf{3 0}$ & 3.41 & 98 & $\mathrm{C} 5 \mathrm{H} 6 \mathrm{O} 2$ & 0.18 & Alcohol Compounds \\
\hline $\mathbf{3 1}$ & 3.63 & 100 & $\mathrm{C} 6 \mathrm{H} 12 \mathrm{O}$ & 14.87 & 2-Furanmethanol \\
\hline
\end{tabular}

\begin{tabular}{|c|c|c|c|c|c|}
\hline 32 & 6.53 & 284 & $\mathrm{C} 19 \mathrm{H} 40 \mathrm{O}$ & 1.32 & n-Nonadecanol-1 \\
\hline 33 & 8.27 & 386 & $\mathrm{C} 26 \mathrm{H} 46 \mathrm{O}$ & 2.42 & Cholesterol \\
\hline 34 & 9.81 & 400 & $\mathrm{C} 28 \mathrm{H} 48 \mathrm{O}$ & 1.31 & Ergost-5-en-3-ol, (3.beta.)- \\
\hline 35 & 10.29 & 412 & $\mathrm{C} 29 \mathrm{H} 48 \mathrm{O}$ & 2.78 & Stigmasterol \\
\hline 36 & 3.81 & 202 & $\mathrm{C} 11 \mathrm{H} 22 \mathrm{O} 3$ & 0.31 & 1-Butanol, 3-methyl-, carbonate $(2: 1)$ \\
\hline 37 & 4.39 & 130 & $\mathrm{C} 8 \mathrm{H} 18 \mathrm{O}$ & 0.12 & 1-Hexanol, 2-ethyl- \\
\hline 38 & 5.16 & 134 & $\mathrm{C} 5 \mathrm{H} 10 \mathrm{O} 4$ & 1.17 & 1,2,3-Propanetriol, 1-acetate \\
\hline 39 & 5.21 & 134 & $\mathrm{C} 5 \mathrm{H} 10 \mathrm{O} 4$ & 0.53 & 1,2,3-Propanetriol, monoacetate \\
\hline & & & 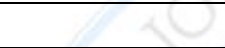 & & Aldehyde and ketone Compounds \\
\hline 40 & 3.85 & 98 & $\mathrm{C} 5 \mathrm{H} 6 \mathrm{O} 2$ & 0.26 & 1,2-Cyclopentanedione \\
\hline 41 & 4.15 & 144 & $\mathrm{C} 6 \mathrm{H} 8 \mathrm{O} 4$ & 0.20 & 2,4-Dihydroxy-2,5-dimethyl-3(2H)-furan-3-one \\
\hline 42 & 4.18 & 102 & $\mathrm{C} 4 \mathrm{H} 6 \mathrm{O} 3$ & 0.30 & 2-Hydroxy-gamma-butyrolactone \\
\hline 43 & 4.88 & 144 & $\mathrm{C} 6 \mathrm{H} 8 \mathrm{O} 4$ & 1.46 & 4H-Pyran-4-one, 2,3-dihydro-3,5-dihydroxy-6-methyl- \\
\hline 44 & 4.94 & 90 & $\mathrm{C} 3 \mathrm{H} 6 \mathrm{O} 3$ & 3.78 & Propanal, 2,3-dihydroxy- \\
\hline 45 & 5.12 & 126 & $\mathrm{C} 6 \mathrm{H} 6 \mathrm{O} 3$ & 1.75 & 2-Furancarboxaldehyde, 5-(hydroxymethyl)- \\
\hline 46 & 6.12 & 162 & $\mathrm{C} 6 \mathrm{H} 10 \mathrm{O} 5$ & 4.94 & 3-Deoxy-d-mannoic lactone \\
\hline & & & 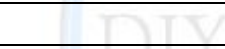 & A & Other Compounds \\
\hline 47 & 3.56 & 330 & $\mathrm{C} 18 \mathrm{H} 18 \mathrm{O6}$ & 0.84 & 4,4'-Bis(methoxymethoxy) benzil \\
\hline 48 & 6.44 & 198 & $\mathrm{C} 12 \mathrm{H} 22 \mathrm{O} 2$ & 1.14 & 2,2'-Bioxepane \\
\hline 49 & 7.16 & 354 & $\mathrm{C} 24 \mathrm{H} 50 \mathrm{O}$ & 0.41 & Dodecane, $1,1^{\prime}$-oxybis- \\
\hline $\mathbf{5 0}$ & 5.78 & 342 & $\mathrm{C} 12 \mathrm{H} 22 \mathrm{O} 11$ & 16.94 & Sucrose \\
\hline 51 & 6.31 & 488 & $\mathrm{C} 35 \mathrm{H} 52 \mathrm{O}$ & 1.91 & $\begin{array}{l}\text { 17-(1,5-Dimethylhexyl)-10,13-dimethyl-3- } \\
\text { styrylhexadecahydrocyclopenta[a]phenanthren-2-one }\end{array}$ \\
\hline 52 & 2.52 & 118 & $\mathrm{C} 7 \mathrm{H} 15 \mathrm{~F}$ & 0.87 & Heptane, 1-fluoro- \\
\hline 53 & 2.59 & 92 & $\mathrm{C} 3 \mathrm{H} 8 \mathrm{O} 3$ & 3.33 & Glycerin \\
\hline 54 & 2.66 & 146 & $\mathrm{C} 8 \mathrm{H} 18 \mathrm{O} 2$ & 1.58 & Propane, 2,2'-[ethylidenebis(oxy)]bis- \\
\hline 55 & 2.72 & 86 & $\mathrm{C} 4 \mathrm{H} 6 \mathrm{O} 2$ & 1.16 & 2,2'-Bioxirane \\
\hline \multicolumn{4}{|c|}{ Total identified compounds } & $84.76 \%$ & 200 \\
\hline
\end{tabular}


Anatomical and Phytochemical Study of Glossostemon bruguieri

(Desf.) Sterculiaceae in Kurdistan Region of Iraq

Khalid Faiq Darweesh

Karzan Mahmood Ahmed

\section{Conclusion}

To the best our information this is the first research of comparative anatomical and phytochemical investigation different parts of G. bruguieri plant, the chemical compositions from the investigated Moghat Plant were rich in different class of organic compounds like hydrocarbons, nitrogens, esters, acids, alcohols, aldehydes, ketones and others, supported by the anatomical study of stem ,inflorescence, leaf and leaf petioles which showed present of Schizogenous secretory ducts of mucilaginous materials. However, further studies are needed for the isolation of individual compounds from the plant extracts of G. bruguieri while in vitro and vivo studies are needed in order to use a natural source for handling different kind of disease such as anti-inflammatory, antimicrobial antioxidant as well as antitumor agent and aphrodisiac uses of different parts of the plant in addition to root.

\section{$\underline{\text { References }}$}

1. Al-Rawi and Chakravarty. (1964). Medicinal plants of Iraq. Baghdad: National Herbarium of Iraq.

2. Beck, C. B. (2010). An Introduction to Plant Structure,Plant Anatomy for the TwentyFirst Century.Second Edition. The Edinburgh Building, Cambridge CB2 8RU, UK: Cambridge University Press.

3. Cheila, D.O.S., Fabricio, M.C., Fabricio, A.O., Evandro, P., Carmen, B.B.F., Susana, M.W.S., Maria, L.S. (2012). Antimicrobial activity [2methacryloyloxy) ethyl] trimethylammonium chloride against Candida spp,1-4.

4. Elija, K., Vaishali, B., Adsul, M.K., Deshpande, N.R. and Kashalkar, R.V. (2012). Antibacterial activity of Dibutyl Phthalate : A secondary metaboli isolated from Ipomoea carnea stem. Journal of Pharmacy Research, 5, No 1.

5. El-Kiey and Hashem,. (1957). A Pharmacognostical study of Egyptian Moghat. Proc. Pharm.soc egypt, 39,12. 
Anatomical and Phytochemical Study of Glossostemon bruguieri

(Desf.) Sterculiaceae in Kurdistan Region of Iraq

Khalid Faiq Darweesh
Karzan Mahmood Ahmed

6. El-Sayed N.H.., A. S.awaad and T.J.Marbry (2004). Phytochemical studies and effect on urine volume of Glossostemon bruguieri. Indian Journal of Experimental Biology, 42, pp. 186-189.

7. El-Sayed M.H. , B.M. Refaat and M. H. Sharaf. (2014). Microbiological evaluation of antibacterial potentiality of some edible plant extracts against multidrug resistant (MDR) human pathogens. International Current Pharmaceutical Journal, 336-339.

8. Evert, R. F. (2006). Esau's plant anatomy. A John Wiley \& Sons, Inc., Publication.

9. Sher, H. and M. N. Alyemeni. (2011). Pharmaceutically important plants used in traditional system of Arab medicine for the treatment of livestock ailments in the kingdom of Saudi Arabia. African Journal of Biotechnology, Vol. 10(45), pp. 9153-9159.

10. Hema, R., Kumaravel, S., and Alagusundaram. (2011). GC/MS Determination of Bioactive components of Murraya koenigii. Journal of American Science, 7(1).

11. Hofreiter M., Seree D., Poinar H.N., Kuch M., and Pabo S. Nature Reviews Genetics (2001) 2:353.

12. Hsouna, A.B., Trigie, M., Mansour, R.B., Jarraya, R.M., Damak, M., and Jaoua, S., (2011). Chemical composition, cytotoxicity effect and antimicrobial activity of Ceratonia silisqua essential oil with preservative effects against listeria inoculated in minced beef meat. International Journal of Food Microbiology, 148(1), 66-72.

13. Ibrahim N, el-Eraky W, el-Gengaihi S, Shalaby AS. (1997). Chemical and biological evaluation of proteins and mucilages from roots and seeds of Glossostemon bruguieri Desf. (Moghat). Plant Foods Hum Nutr, 50(1):55-61.

14. IUCN. (2012). IUCN Red List Categories and Criteria: Version 3.1. Second edition.Gland, Switzerland and Cambridge, UK: IUCN. iv + 32pp.

15. Margareth B. C. Gallo., Miranda J. Sarachine (2009). Biological activates of Lupeol. International journal of biomedical and pharmaceutical sciences 3 (special issue 1): 4666.

16. Meselhy, Meselhy R. (2003). Constituents from Moghat, the Roots of Glossostemon bruguieri(Desf.). molecules, 614-621. 
Anatomical and Phytochemical Study of Glossostemon bruguieri

(Desf.) Sterculiaceae in Kurdistan Region of Iraq

\section{Khalid Faiq Darweesh Karzan Mahmood Ahmed}

17. Eddouks M and NA. Zeggwagh. (2014). A Short Review of Some Medicinal Plants And Phytocompounds With Hypotensive And Hypoglycemic Activities. International Journal of Diabetology \& Vascular Disease Research, 63-66.

18. Townsend C.C..and Evan Guest. (1980). Flora of Iraq. (Vol. Four part one). Baghdad, Iraq: Ministry of Agriculture .

19. Venkata, R., Samuel, L., Pardha, S.M., Narashimha, R. Naga, V.K.A., Sudhakar, M., and Radhakrishnan, T.M. (2012). Antibacterial, antioxidant activity and GC-MS Analysis of Eupatorium odoratum. Asian J. Pharm. Clin. Res., 5. Suppl 2, 0974-2441.

20. William Marias Malisoff (1943). Dictionary of bio-chemistry and related subjects. Philosophical library. Pp. 311, 350, 573. ASIN B0006AQ0NU.

21. Zain M. E. ,A. S. Awaad M. R. Al-Othman and S. K. Aldosary (2014). Antibacterial , Antifungal and phytochemical analysis of some deser plant against human pathogenic bacteria and fungi. Life science journals, 343-349. 